\title{
Clinical outcome of lower esophageal sphincter- and vagus-nerve- preserving partial cardiectomy for early gastric cancer of the subcardia
}

\author{
Hideo Matsumoto $\cdot$ Haruaki Murakami • \\ Hisako Kubota $\cdot$ Masaharu Higashida $\cdot$ \\ Masafumi Nakamura $\cdot$ Toshihiro Hirai
}

Received: 20 September 2012/ Accepted: 18 May 2014/Published online: 7 June 2014

(c) The International Gastric Cancer Association and The Japanese Gastric Cancer Association 2014

\begin{abstract}
Background No definitive operative method has been established for the treatment of early subcardial gastric cancer. Our newly developed technique involves local resection of the subcardia while preserving the lower esophageal sphincter and vagus nerve. A new fornix is constructed to accept the transposed esophagus.

Methods Thirty patients underwent this procedure between July 2003 and December 2010. Continuous gastric pH monitoring was performed immediately after surgery, and esophageal manometry was undertaken 1 month later. Serum total protein, albumin, total cholesterol, cholinesterase, and body mass index (BMI) were recorded every 3 months. Pre- and postoperative oral intake were compared, reflux symptoms were recorded, and reflux esophagitis was assessed by endoscopy after 1 year.

Results Twenty-five patients (86\%) reported no symptoms of reflux, and $27(92.8 \%)$ patients could eat $70 \%$ or more of what they had eaten before surgery. Lower esophageal pressures were found to be $>10 \mathrm{mmHg}$ in $66.7 \%$ of patients, and the fraction of time that $\mathrm{pH}<4$ was $<5 \%$ of the 24-h monitoring period in $70 \%$. Serum parameters and BMI were unchanged.

Conclusions This surgical technique is a useful means of preserving postoperative quality of life after local gastrectomy by preventing reflux and maintaining nutritional status.
\end{abstract}

H. Matsumoto $(\square) \cdot$ H. Murakami · H. Kubota · M. Higashida . M. Nakamura - T. Hirai

Department of Digestive Surgery, Kawasaki Medical School,

577 Matsushima, Kurashiki, Okayama 701-0192, Japan

e-mail: h-matsu@med.kawasaki-m.ac.jp
Keywords Early gastric cancer of subcardia $\cdot$ Lower esophageal sphincter - Nerve preservation .

Esophagogastrostomy $\cdot$ Reconstruction

\section{Introduction}

There is substantial debate regarding the best means of treating early subcardial gastric cancer. Mucosal lesions of the subcardia are usually resected endoscopically, but total gastrectomy (TG) has traditionally been used for invasive lesions of the submucosa and/or residual cancer remaining after endoscopic resection. Some believe that TG is excessive under these circumstances and advocate proximal gastrectomy (PG) as a more conservative approach.

Total gastrectomy is a relatively straightforward procedure, but preventing reflux and maintaining nutritional status can be challenging after the operation. Some investigators have concluded that TG is superior to PG in terms of gastroesophageal reflux [1-3], despite the fact that an esophagogastric anastomosis would not be expected to prevent reflux. Conflicting reports suggest that interposition of a segment of jejunum as part of a PG resulted in fewer clinical signs and symptoms $[4,5]$.

The optimal reconstruction method after PG is yet to be established, mainly because postoperative symptoms are strongly influenced by surgical technique. The two most important issues affecting quality of life after surgery for cancer of the gastric cardia are esophageal reflux due to disruption of the antireflux mechanism (which includes the lower esophageal sphincter, LES [3], and the vagus nerve) and preservation and peristalsis of the remnant stomach.

We reported a new method of treating subcardial cancers of depth T1b by partial cardiectomy in a series of 6 
cases in 2006 [6]. Here we describe our experience of a modified technique in a further series of 30 cases.

\section{Materials and methods}

Indication of operation

We applied this technique to tumors clinically diagnosed as T1 N0 and located around the lesser curvature of the upper third of the stomach. The proximal margin of the tumor had to be $2.0 \mathrm{~cm}$ away from the squamocolumnar junction. We did not set a limit on the tumor size, but we selected those in which at least half of the stomach could be preserved after resection.

Operative technique

After fenestration of the gastrohepatic ligament through an upper midline incision, the hepatic branches of the anterior vagus nerve are identified and followed proximally to confirm the location of the gastric branches. Only the anterior gastric branches are then cut. The posterior branch of the vagus nerve is identified on entry to the retroperitoneum on the right side of the crus of the diaphragm. The lymph nodes along the celiac and splenic arteries are dissected, preserving the posterior branches of the vagus nerve. The gastric branches are cut after the structure has been clearly confirmed, preserving the celiac branch.

Following dissection of the lymph nodes along the lesser curvature and left paracardial portion of the stomach, two or three branches of the upper short gastric vessels are incised to create the new fornix, the primary fornix being anastomosed to the distal edge of the subsequent gastrectomy. The esophagogastric (EG) junction is dissected, preserving the LES and the structures around the EG junction (for example the phrenoesophageal ligament). In our experience, the EG junction tends to be just below the last branch of the gastric vein [7]. The esophagus is cut at the level of this vein, and the squamocolumnar junction is confirmed by staining the mucosa with Lugol's solution, the intention being to leave no gastric mucosa. Figure 1 shows the local anatomy [8]. The lesion can then be excised through an incision in the greater curvature with minimum 2-cm margins under direct vision. The resection margin and the esophagus and anterior wall of the remnant stomach are anastomosed by hand (Fig. 2). In our first report, the anastomotic site of the esophagogastrostomy was distal to the closure line, but we changed the anastomosis to the proximal site of the closure line, as we found this to be a technically easier and more effective means of creating the new fornix. Moreover, we found that the finger bougie used to create the pyloric ring was unnecessary.

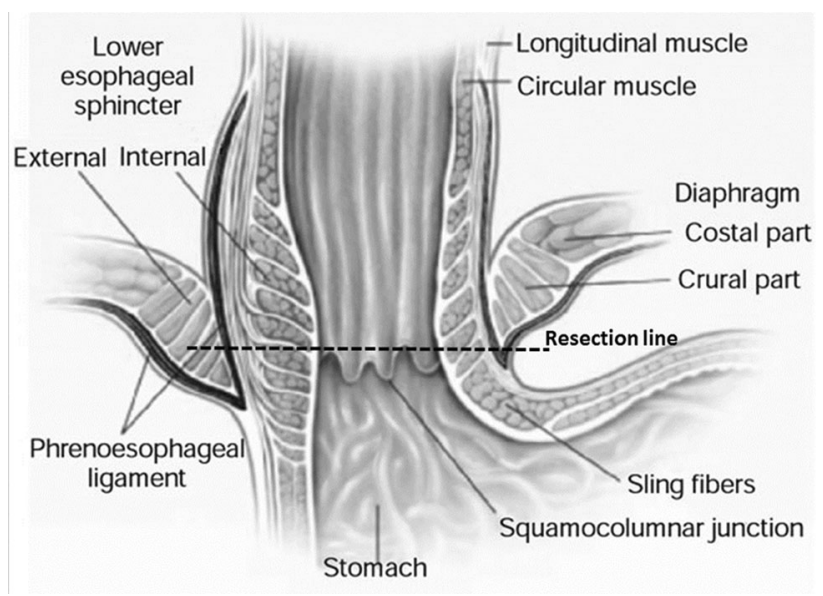

Fig. 1 Anatomy of the esophagogastric junction [8]

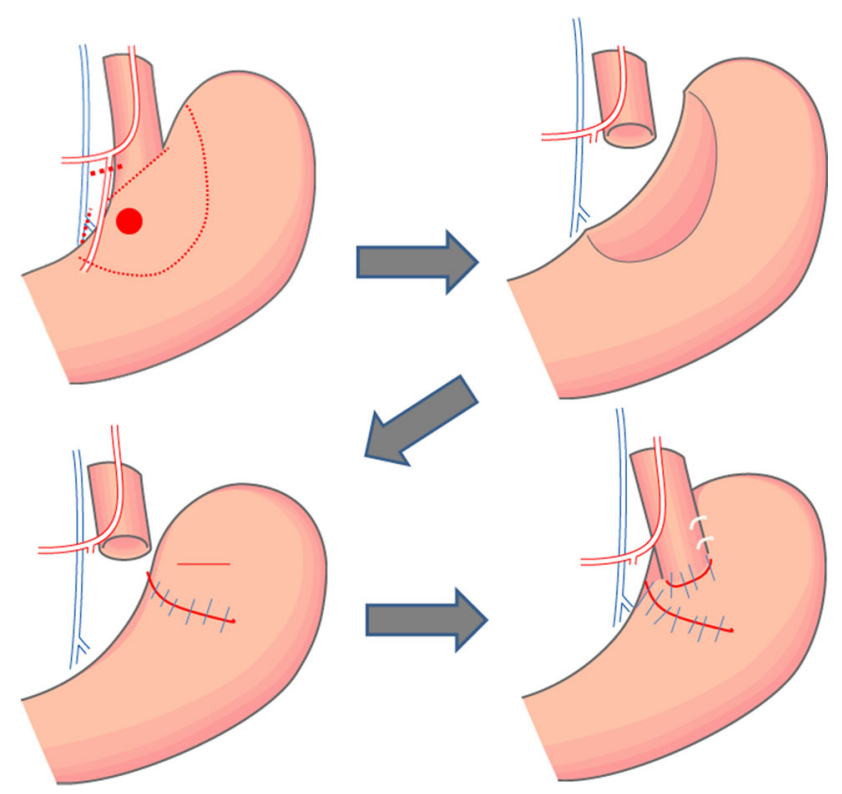

Fig. 2 Illustration of the procedure

Postoperative assessment

A 24-h pH monitor (Digitrapper; Medtronic Inc., Shoreview, MN, USA) was used 1 month after surgery, along with a simultaneous evaluation based on esophageal manometry (InSIGHT G3; Sandhill Scientific Inc., Highlands Ranch, CO, USA). Reflux symptoms and oral intake were determined in an interview 1 year postoperatively. Serum total protein, albumin, total cholesterol, and cholinesterase were measured and body mass index (BMI) was recorded at 1, 3, 6, and 12 months. Endoscopy was performed 1 year postoperatively. The study protocol was approved by the Institutional Review Board of Kawasaki Medical School. All cancers were classified according to 
the seventh edition of the International Union Against Cancer UICC-TNM classification [9].

Statistical analysis

Survival curves were plotted according to the KaplanMeier method. Statistical calculations were performed using JMP 8 statistical software (SAS Institute, Inc. Cary, NC, USA). Statistical significance was defined as $p<0.05$.

\section{Results}

Between July 2003 and December 2010, 653 patients were treated in our department for gastric cancer; 602 of these underwent gastrectomy. Thirty patients with stage $\mathrm{T} 1 \mathrm{~b}$ disease and no lymph node metastasis underwent our procedure, having given full informed consent. Nine patients had undergone endoscopic mucosal resection or endoscopic submucosal dissection before the operation. Four were found to have tumor in the dissection margins and the others had extensive tumor invasion into the submucosal layer. All the tumors were in the subcardia of stomach around the lesser curvature. We used our method for 31 lesions in 30 patients with no mortality or morbidity. One patient had a lesion of both the subcardia and the middle of the body at the lesser curvature.

The mean tumor size was $26.8 \mathrm{~mm}$ (ranged 11-65 mm), and the mean stomach resection area was $82.5 \times 62.0 \mathrm{~mm}$ (ranged $44 \times 32$ to $120 \times 80 \mathrm{~mm}$ ). The histological depth of tumor invasion was classified as stage T1a in 6 cases, $\mathrm{T} 1 \mathrm{~b}$ in 23 cases, and T2 in 1 case. The tumors were located in the upper area of the lesser curvature in 16 cases, the posterior side of the upper area in 12 cases, the anterior side of the upper area in 2 lesions, and the middle area of the lesser curvature in 1 case (Table 1). Metastasis to a single lymph node was identified on histological examination in two cases. The mean operation time was $200 \mathrm{~min}$, and the mean intraoperative blood loss was $363 \mathrm{~mL}$.

Twenty-four patients underwent $\mathrm{pH}$ monitoring. Four patients could not be examined because of a machine malfunction, 1 patient was being treated for another illness, and 1 patient refused $\mathrm{pH}$ monitoring. Manometry was performed in 28 patients. One patient was being medicated for a concurrent illness, and another refused the test. Symptoms of reflux and assessment of oral intake were determined at interview for 26 patients: 3 patients were being treated at another hospital and another refused the offer of an interview. Blood tests were taken and BMI recorded in 26 patients, and endoscopy was performed in 28 patients.

Twenty-four patients (92\%) did not have reflux symptoms, and 25 patients $(92.2 \%)$ could eat $70 \%$ or more of
Table 1 Characters of the cancer

\begin{tabular}{lll}
\cline { 2 - 2 } & Depth & \\
& T1a & 6 \\
T1b & T2 & 25 \\
& Lymph node metastasis \\
& $\mathrm{N} 0$ & 28 \\
& $\mathrm{~N} 1$ & 2 \\
& Endoscopic therapy & \\
& EMR & 6 \\
EMR endoscopic mucosal & ESD & 2 \\
resection, ESD endoscopic sub- & Location & $($ lesions $)$ \\
mucosal dissection, $U$ upper & $\mathrm{U}$, less & 16 \\
hird of stomach, $M$ middle third & $\mathrm{U}$, post & 12 \\
of stomach, less lesser curva- & $\mathrm{U}$, ant & 2 \\
ure, post posterior wall, ant & $\mathrm{M}$, less & 1 \\
anterior wall & &
\end{tabular}

what they had eaten preoperatively. There were no significant changes in BMI postoperatively (Fig. 3). Nineteen of 28 patients $(67.8 \%)$ had LES pressures $>10 \mathrm{mmHg}$. Seventeen of 24 patients $(70.8 \%)$ had a fraction time $\mathrm{pH}$ of $<4$ for $<5 \%$ of the 24 -h pH-monitoring period. In all cases where the LES pressure had been preserved within the normal range, 24-h $\mathrm{pH}$ monitoring was also normal. In some cases, the 24-h pH monitoring was normal despite reduced LES pressures; however, in the cases where reflux was detected by 24-pH monitoring, LES pressures were found to be low at endoscopy.

All patients underwent a videofluoroscopic swallowing examination on the seventh postoperative day, confirming normal antral peristalsis and no leakage or reflux to the esophagus with the patient in the left decubitus position and the head at a low level.

Endoscopic findings revealed that 5 patients exhibited reflux esophagitis: grade A and B in two, grade $\mathrm{C}$ in one, and grade $\mathrm{D}$ in one according to the Los Angeles Classification of Reflux Esophagitis [10] (Table 2). The remaining 24 patients had no reflux esophagitis. Serum nutritional status (total protein, albumin, total cholesterol, and cholinesterase) did not change postoperatively (Fig. 4).

Two patients required balloon dilatation for an anastomotic stricture. These patients were found to have low LES pressures and high rates of reflux on $\mathrm{pH}$ monitoring. In 4 patients, other cancers were found 4-6 years later; three in the lower stomach were treated by endoscopic resection, and one was treated by total gastrectomy, as the extent of the lesion in the antrum could not be accurately delineated on endoscopy. One patient who had been found to have invasion to the $\mathrm{T} 1 \mathrm{~b}$ layer without vascular involvement or lymph node metastasis died of hepatic metastases 2 years after the operation. The overall 5-year survival rate was 
Fig. 3 Symptoms and changes in BMI

\begin{tabular}{|c|c|c|c|c|}
\hline $\begin{array}{c}\text { Reflux symptom } \\
(\mathrm{n}=26)\end{array}$ & \multicolumn{2}{|c|}{ No } & \multicolumn{2}{|c|}{ Yes (Severe) } \\
\cline { 2 - 5 } & \multicolumn{2}{|c|}{$\begin{array}{c}24 \\
(92 \%)\end{array}$} & \multicolumn{2}{c|}{$\begin{array}{c}2(1) \\
(6.8 \%(3.4 \%)\end{array}$} \\
\hline \multirow{2}{*}{$\begin{array}{c}\text { Oral Intake } \\
(\mathrm{n}=26)\end{array}$} & $90-100 \%$ & $70-80 \%$ & $50-60 \%$ & $<50 \%$ \\
\cline { 2 - 5 } & $\begin{array}{c}2 \\
(7.6 \%)\end{array}$ & $\begin{array}{c}22 \\
(84.6 \%)\end{array}$ & $\begin{array}{c}1 \\
(4.1 \%)\end{array}$ & $\begin{array}{c}1 \\
(4.1 \%)\end{array}$ \\
\hline
\end{tabular}

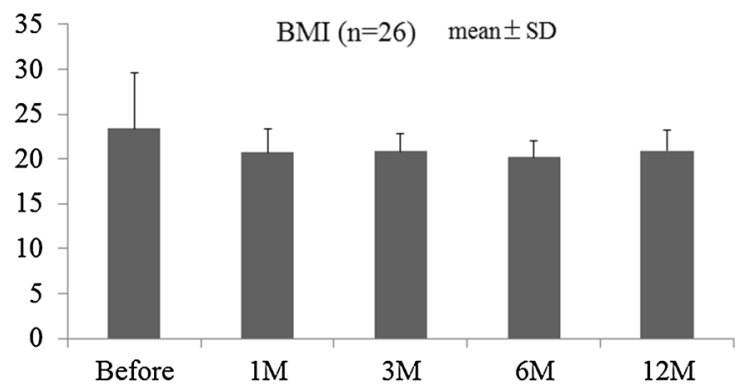

Table 2 Manometry, 24-h pH monitoring, and endoscopic findings

Mean LESP $(\mathrm{mmHg})(n=28)$

\begin{tabular}{llll}
\hline LESP $\leq 5$ & $5<$ LESP $<10$ & $10<$ LESP $<15$ & LESP $>15$ \\
\hline $2(7.1 \%)$ & $7(25.0 \%)$ & $6(21.4 \%)$ & $13(46.4 \%)$
\end{tabular}

Fraction of time $\mathrm{pH}<4(n=24)$

\begin{tabular}{|c|c|c|c|}
\hline $\begin{array}{l}0-5 \% \\
17(70.8 \%)\end{array}$ & & $\begin{array}{l}5-10 \% \\
2(8.3 \%)\end{array}$ & $\begin{array}{l}10 \%> \\
5(20.8 \%)\end{array}$ \\
\hline L.A. classification & Normal & Grades A, B & Grades C, D \\
\hline \multicolumn{4}{|c|}{ Endoscopic findings after one year } \\
\hline Case $(n=28)$ & 24 & 2 & 2 \\
\hline
\end{tabular}

$96.5 \%$. The median follow-up period was 62.6 months (range 19.6-120.2 months); 2 patients were lost to followup at 56.8 and 65.5 months.

\section{Discussion}

We found that our new method is superior to other means of reconstruction in two ways. First, it is simple and prevents reflux while preserving the structure and function of the LES. Second, it enables continued oral intake, as partial resection and reshaping by hand sewing preserves a large portion of the stomach. Anastomosis to the anterior wall of the stomach is shown in Fig. 2. A new fornix is created and

\section{Serum nutritional status $(n=26)$}

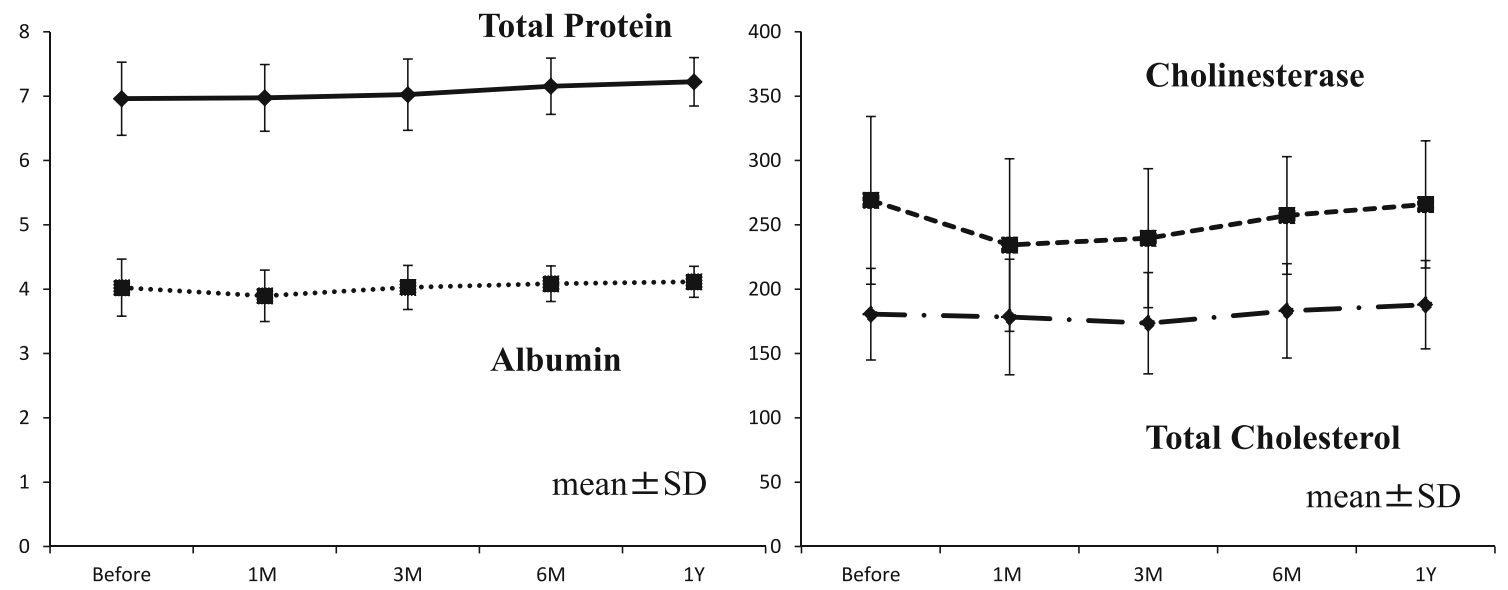

Fig. 4 Changes in serum nutritional status 
moderate peristalsis can be observed in the antrum on postoperative videofluoroscopy examinations, likely because the hepatic branch (including the pyloric branch) of the vagus nerve is preserved.

The choice of reconstruction technique is crucial for maintaining the postoperative quality of life of patients with cancers in the upper third of the stomach. Takiguchi et al. [11] reported the outcomes of a series of 586 patients treated for early gastric cancer in the upper third of the stomach in 19 hospitals in Japan over 9 years. Total gastrectomy was undertaken more frequently than proximal gastrectomy (76.3 versus $21.8 \%$, respectively). For reconstruction after PG, esophagogastrostomy and jejunal interposition were performed in $50(39.1 \%)$ and 35 $(27.3 \%)$ patients, respectively.

Several papers have compared esophagogastrostomy and jejunal interposition. Tokunaga et al. [12] reported that esophagogastrostomy is superior to jejunal interposition after proximal gastrectomy when subjective symptoms are evaluated. Early and late dumping syndromes were observed equally as often in the groups, as were symptoms of gastroesophageal reflux; however, abdominal discomfort after meals, continuous gastric fullness, and hiccups between meals were reported significantly more often by those with jejunal interpositions. There was no significant difference in change in body weight between the groups. In this series, esophagogastrostomy with preservation of the hepatic and celiac branches of the vagus nerve was associated with a high incidence of severe esophagitis (greater than grade D esophagitis was found at endoscopy in $22.2 \%$ of the cases), presumably due to the lack of an anatomical structure to prevent esophageal reflux [13]. A similar proportion was reported in a smaller series using the same technique (5 of 20 patients, $25.0 \%$ ) [14]. In contrast, Tokunaga et al. [13] previously reported that jejunal interposition helps to prevent reflux esophagitis after PG. The reported incidence has varied between 3 and $78 \%$, although in some reports the method of evaluation has not been clear [3, 13-15]. The incidence of reflux esophagitis was substantially lower in our series.

In 1989, Yan advocated preservation of the LES as a useful means of preventing esophagogastric reflux in dogs [16]. Hirai et al. [7] first reported that preservation of the LES during total gastrectomy prevented reflux esophagitis in 1995. Tomita et al. stated that "preservation of the LES and pyloric sphincter in near total gastrectomy reconstructed by jejunal interposition" improved the quality of life of patients with early gastric cancer [17, 18]. We adopted this method for esophagogastrostomy in 2005 [6].

Postoperative quality of life after a PG with jejunal interposition has been evaluated, and the technique is also considered beneficial for preventing reflux esophagitis [1923], albeit in small series. Objective outcome measures- such as endoscopic findings or 24-h pH monitoring-were reported, and the incidences of subjective symptoms were rarely compared [19, 21, 24].

Our technique uses hand sewing to better preserve the LES, which can be damaged by instrumentation. We also cut the esophagus at the EG junction, guiding the terminal branch of the left gastric vein across the uppermost portion of the stomach and staining with Lugol's solution to confirm that there is no residual gastric mucosa. Reconstruction with mechanical anastomosis may have been possible, but we had no experience or data to support this. Some $[12-14,17,18]$ have reported the use of mechanical anastomosis for esophagojejunostomy, but have not measured the postoperative function of the LES. Hand-sewing the anastomosis may help to prevent reflux; however, we believe that our finding that normal LES pressures are associated with a lower incidence of reflux symptoms demonstrates that preserving the LES and the surrounding structures is crucial. In our opinion, the additional suturing needed to create the angle of His is not necessary.

All lesions in our study were classified as carcinomas of the esophagogastric junction (EGJ) according to the seventh edition of the UICC-TNM classification [9]. We excluded lesions that contacted the EG junction, as adequate safety margins could not be ensured while preserving the LES. The safety margin was considered to be $>1.5 \mathrm{~cm}$ distant from the macroscopic tumor margin in cases with negative lymph node metastasis [7]. We recommend that the tumor should be resected with a safety margin of $>2 \mathrm{~cm}$, and that the margins should always be examined in frozen sections by a pathologist during the procedure.

It is essential to maintain surveillance of the remnant stomach because of the significant risk of metachronous cancer. Iwata et al. [21] reported that it was easier to enter the remnant stomach and duodenum with an endoscope in patients with a jejunal interposition if a pouch had been formed. In our series, we found postoperative surveillance endoscopy straightforward to perform, and detected remnant stomach cancers in four patients (13.3\%), even after Helicobacter pylori eradication. All metachronous tumors were found in the lower stomach and were cured; by endoscopic dissection in 3 patients and resection of the remnant stomach in the other. One patient died of liver metastases 1 year after the operation, but this death was not a consequence of the surgical technique.

We had no morbidity and mortality in the hospital stay, but 2 cases of anastomosis stricture occurred during the period from the surgery to $>6$ months after it. These strictures were due to reflux esophagitis and improved following one balloon dilatation with administration of a proton pump inhibitor.

In those patients who complained of gastroesophageal reflux, proton pump inhibitors were used in 4 patients for 
1 year. Reflux symptoms improved with time, perhaps as a result of improved peristalsis in the antrum, pylorus, and duodenum.

In our first report of this method [6], we stated that we closed the resection line of the stomach longitudinally. In the present work, we used a horizontal closure to create a better stomach shape. We also abandoned the use of the finger bougie technique at the pylorus, as we considered it to be unnecessary if the pyloric branch of the vagus nerve is preserved. Also, as the pylorus was preserved, no patients complained of the symptoms of dumping syndrome. All patients who underwent this procedure were satisfied with their postoperative quality of life.

\section{Conclusion}

We found that our surgical technique was convenient and helped to maintained patients' postoperative quality of life. It also permitted straightforward surveillance of the remnant stomach. We believe that this method is a useful option for the treatment of early subcardial gastric cancer.

Conflict of interest None of the authors have any financial or other conflicts of interest to declare.

\section{References}

1. Katsoukis IE, Roboti JF, Kouraklis G, Yannopoulos PA. What is the difference between proximal and total gastrectomy regarding postoperative bile reflux into the oesophagus? Dig Surg. 2006;23:325-30.

2. Ji YA, Ho GY, Min GC, Jae HN, Tae SS, Sung K. The difficult choice between total and proximal gastrectomy in proximal early gastric cancer. Am J Surg. 2008;196:587-91.

3. Nozaki I, Hato I, Kobatake T, Ohta K, Kubo Y, Kurita A. Longterm outcome after proximal gastrectomy with jejunal interposition for gastric cancer compared with total gastrectomy. World $\mathbf{J}$ Surg. 2013;37:558-64.

4. Takeshita K, Sekita Y, Tani M. Medium- and long-term results of jejunal pouch reconstruction after a total and proximal gastrectomy. Surg Today. 2007;37:754-61.

5. Hsu CP, Chen CY, Hsieh YH, Hsia JY, Shai SE, Kao CH. Esophageal reflux after total or proximal gastrectomy in patients with adenocarcinoma of the gastric cardia. Am J Gastroenterol. 1997;92:1347-50.

6. Hirai T, Matsumoto H, Ikial K, Hirabayashi Y, Kawabe Y, Ikeda $\mathrm{M}$, et al. Lower esophageal sphincter- and vagus-preserving proximal partial gastrectomy for early cancer of the gastric cardia. Surg Today. 2006;36:874-8.

7. Hirai T, Saeki S, Matsuki K, Yamashita Y, Iwata T, Yoshimoto A, et al. Preservation of the lower esophageal sphincter during total gastrectomy for gastric cancer to prevent postoperative reflux esophagitis. Surg Today. 1995;25:507-14.

8. Ravinder KM, Raj KG. Sphincter mechanisms at the lower end of the esophagus. GI Motility Online. 2006. doi:10.1038/gimo14. URL: http://www.nature.com/gimo/contents/pt1/full/gimo14.html.
9. Sobin LH, Gospodarowics MK, Wittekind $\mathrm{CH}$. International Union Against Cancer. TNM classification of malignant tumors. 7th ed. Hoboken: Wiley; 2009. p. 69-73.

10. Armstrong D, Bennett JR, Blum AL, Dent J, De Dombal FT, Galmiche JP, et al. The endoscopic assessment of esophagitis: a progress report on observer agreement. Gastroenterology. 1996;111:85-92.

11. Takiguchi S, Masuzawa T, Hirano M, Imamura H, Kimura Y, Fujita $J$, et al. Pattern of surgical treatment for early gastric cancers in upper third of the stomach. Hepatogastroenterology. 2011;58:1823-7.

12. Tokunaga M, Hiki N, Ohyama S, Nunobe S, Miki A, Fukunaga T, et al. Effects of reconstruction methods on a patient's quality of life after a proximal gastrectomy: subjective symptoms evaluation using questionnaire survey. Langenbecks Arch Surg. 2009;394:637-41.

13. Tokunaga M, Ohyama S, Hiki N, Hoshino E, Nunobe S, Fukunaga $T$, et al. Endoscopic evaluation of reflux esophagitis after proximal gastrectomy: comparison between esophagogastric anastomosis and jejunal interposition. World J Surg. 2008;32: 1473-7.

14. Ichikawa D, Komatsu S, Okamoto K, Shiozaki A, Fujiwara H, Otsuji E. Evaluation of symptoms related to reflux esophagitis in patients with esophagogastrostomy after proximal gastrectomy. Langenbecks Arch Surg. 2013;398:697-701.

15. Seshimo A, Miyake K, Amano K, Aratake K, Kameoka S. Clinical outcome of esophagogastrostomy after proximal gastrectomy for gastric cancer. Hepatogastroenterology. 2013;60: 616-9.

16. Yan Y. Experimental study of antigastroesophageal reflux by preservation of the lower esophageal sphincter. Zhonghua Wai Ke Za Zhi. 1989;27:121-3.

17. Tomita R, Kurosu Y, Takizawa H, Abe Y. Pathophysiological assessments in lower esophageal sphincter and pylorus preserving nearly total gastrectomy with a single jejunal interposition. J Jpn Coll Surg. 1996;21:700.

18. Tomita R, Shigeru F, Tanjoh K, Fukuzawa M. Operative technique on nearly total gastrectomy reconstructed by interposition of jejunal $\mathbf{J}$ pouch with preserving of vagal nerve, lower esophageal sphincter, and pyloric sphincter for early gastric cancer. World J Surg. 2001;25:1524-31.

19. Shiraishi N, Hirose R, Morimoto A, Kawano K, Adachi Y, Kitano S. Gastric tube reconstruction prevented esophageal reflux after proximal gastrectomy. Gastric Cancer. 1998;1:78-9.

20. Adachi Y, Inoue T, Hagino Y, Shiraishi N, Shimoda K, Kitano S. Surgical results of proximal gastrectomy for early-stage gastric cancer: jejunal interposition and gastric tube reconstruction. Gastric Cancer. 1999;2:40-5.

21. Iwata T, Kurita N, Ikemoto T, Nishioka M, Andoh T, Shimada M. Evaluation of reconstruction after proximal gastrectomy: prospective comparative study of jejunal interposition and jejunal pouch interposition. Hepatogastroenterology. 2006;53:301-3.

22. Uyama I, Ogiwara H, Takahara T, Kikuchi K, Iida S. Laparoscopic and minilaparotomy proximal gastrectomy and esophagogastrostomy: technique and case report. Surg Laparosc Endosc. 1995;5:487-91.

23. Kameyama J, Ishida H, Yasaku Y, Suzuki A, Kuzu H, Tsukamoto M. Proximal gastrectomy reconstructed by interposition of a jejunal pouch. Surgical technique. Eur J Surg. 1993;159:491-3.

24. Tomita R, Fujisaki S, Tanjoh K, Fukuzawa M. A novel operative technique on proximal gastrectomy reconstructed by interposition of a jejunal $\mathrm{J}$ pouch with preservation of the vagal nerve and lower esophageal sphincter. Hepatogastroenterology. 2001;48: $1186-91$. 